\title{
Structural neuroanatomy of face processing in frontotemporal lobar degeneration
}

\author{
Rohani Omar, Jonathan D Rohrer, Julia C Hailstone, Jason D Warren
}

- Additional materials are published online only. To view these files please visit the journal online (http://jnnp.bmj. com)

Dementia Research Centre, Institute of Neurology, University College London, London, UK

\section{Correspondence to}

Dr J D Warren, Dementia Research Centre, Institute of Neurology, University College London, 8-11 Queen Square, London WC1N 3BG, UK: jwarren@drc.ion.ucl.ac.uk

Received 24 August 2010 Revised 2 November 2010 Accepted 17 November 2010 Published Online First 16 December 2010

\begin{abstract}
Impairments of face processing occur frequently in frontotemporal lobar degeneration (FTLD) but the neuroanatomical basis for these deficits has seldom been studied systematically. Here a prospective voxel based morphometry study is described addressing the neuroanatomy of two key dimensions of face processing - face identification and facial emotion recognition-in a single cohort of 32 patients with FTLD (19 with frontal variant and 13 with temporal variant FTLD). For the FTLD group as a whole, face identification was positively associated with grey matter in the right anterior fusiform gyrus while recognition of angry expressions was positively associated with grey matter in the bilateral insula cortex. FTLD provides a perspective on the neuroanatomy of face processing that is complementary to focal lesion and normal functional imaging work.
\end{abstract}

\section{INTRODUCTION}

Frontotemporal lobar degeneration (FTLD) is a clinically and pathologically diverse group of neurodegenerative diseases associated with focal frontal and temporal lobe atrophy. ${ }^{1}$ Behavioural disturbances are common in FTLD, most saliently in the canonical syndromes of behavioural variant frontotemporal dementia (bvFTD) and semantic dementia (SD). Behavioural change may be partly attributable to defective processing of social signals: in particular, impaired processing of information about human faces is an important clinical issue in both bvFTD and SD. However, the nature of this impairment is likely to differ between the syndromes. SD is characterised by panmodal breakdown of knowledge about objects, concepts and persons, leading to impaired processing of facial identity; progressive prosopagnosia has been associated particularly with selective right temporal lobe atrophy. ${ }^{2-4}$ In contrast, bvFTD is characterised by decline in interpersonal and executive skills, with loss of emotional responsivity and impaired recognition of emotional facial expressions. ${ }^{5} 6$ This differential involvement of face processing functions may assist in early clinical diagnosis and differentiation of SD and bvFTD. ${ }^{4}$ Anatomically, the frontal and temporal cortical areas affected in FTLD overlap brain regions implicated in face processing from focal lesion studies and functional imaging work in healthy subjects, including the anterior and inferior temporal lobes. ${ }^{7}$ However, few studies have addressed systematically the neuroanatomical basis of face processing deficits in FTLD using unbiased techniques such as voxel based morphometry (VBM).
Here we investigated prospectively the structural neuroanatomical substrates of two key dimensions of face processing-identification of familiar faces and recognition of facial emotions-using VBM in a single cohort of patients with FTLD. Based on previous work in patients with focal brain lesions and in healthy subjects, we hypothesised that face identification would correlate with grey matter in inferior temporal regions ${ }^{7}$ while processing of facial emotions would correlate with grey matter in temporal, inferior frontal and insula regions, with partially separable anatomical associations for different facial emotions. ${ }^{8}$

\section{METHODS}

Thirty-two patients fulfilling consensus clinical criteria for FTLD $^{1}$ were recruited. All patients had a general neuropsychological assessment and volumetric brain MRI. The study cohort comprised 19 patients with predominant frontal lobe atrophy (frontal variant (fv) FTLD), each fulfilling clinical criteria for bvFTD ${ }^{1}$; and 13 patients with predominant asymmetric (left or right) temporal lobe atrophy (temporal variant (tv) FTLD), 12 fulfilling clinical criteria for SD (semantic progressive aphasia) ${ }^{1}$ and one (with predominantly right-sided temporal lobe atrophy) presenting with progressive prosopagnosia. Patients were compared neuropsychologically with a group of 22 age matched healthy controls recruited via the Dementia Research Centre database. Subject characteristics are summarised in table 1. Informed consent was obtained in each case and the study was approved by the local research ethics committee in accord with the Declaration of Helsinki guidelines.

All patients were assessed on tests of face identification, affective processing and perceptual analysis (table 1). Face identification was assessed using a Famous Faces Test requiring recognition of black and white photographs of 12 contemporary public figures widely familiar to UK residents (regularly updated from Warrington and James ${ }^{9}$ ). Identities of the individuals in the test (comprising six prominent British politicians, two prominent American politicians, two high profile entertainers and two members of the British Royal Family) are listed in supplementary table 1 (available online only). Evidence of recognition other than naming can be used to score performance on this test, making it suitable for use in individuals with naming impairments (eg, in SD). Facial emotion recognition was assessed using 24 faces from the Ekman and Friesen battery. ${ }^{10}$ Perceptual analysis of faces was assessed using the Benton Facial Recognition Test. ${ }^{11}$ Details of all neuropsychological tests used are provided in the supplementary material (available online only). 
Table 1 Summary of clinical and neuropsychological characteristics

\begin{tabular}{|c|c|c|c|}
\hline & $\begin{array}{l}\text { fvFTLD } \\
(n=19)\end{array}$ & $\begin{array}{l}\text { tvFTLD } \\
(n=13)\end{array}$ & $\begin{array}{l}\text { Controls } \\
(\mathrm{n}=22)\end{array}$ \\
\hline \multicolumn{4}{|l|}{ Demographic data } \\
\hline Gender (M:F) & $15: 4$ & $6: 7$ & 11:11 \\
\hline Handedness ( $R: L)$ & $17: 2$ & $12: 1$ & NA \\
\hline Age (years) & $66.7(8.7)$ & $63.5(8.8)$ & $65.5(8.5)$ \\
\hline \multicolumn{4}{|l|}{ Behavioural data } \\
\hline MMSE & $27.3(3.0)$ & $24.1(3.7) \ddagger$ & NA \\
\hline Benton face perception $(/ 56)$ & $44.9(7.0) \dagger$ & $46.1(4.4) \dagger$ & $48.4(2.6)$ \\
\hline Famous Faces identification (/12) & $11.1(1.5)$ & $7.9(4.5)^{*} \neq$ & NA \\
\hline Ekman emotion recognition (/24) & $17.7(4.8) \dagger$ & $18.1(3.4) \dagger$ & $21.4(1.9)$ \\
\hline \multicolumn{4}{|l|}{ Individual emotions (\% correct) } \\
\hline Anger & $60.5(29.2)$ & $62.5(23.5)$ & $89.5(12.7)$ \\
\hline Disgust & $73.7(30.6)$ & $66.1(31.9)$ & $96.1(12.5)$ \\
\hline Fear & $44.7(34.9)$ & $62.5(27.3)$ & $68.4(27.4)$ \\
\hline Happiness & $94.7(13.4)$ & $91.1(12.4)$ & $100.0(0.0)$ \\
\hline Sadness & $78.9(28.0)$ & $83.9(23.2)$ & $85.5(20.9)$ \\
\hline Surprise & $89.5(24.0)$ & $85.7(25.4)$ & $96.1(9.4)$ \\
\hline Object Decision Test (/20) & $17.2(1.9)$ & $16.9(3.1)$ & $17.5(2.3)$ \\
\hline Synonyms Test (/25) & $21.9(3.3) \dagger$ & $17.4(6.1) \dagger \ddagger$ & $26.1(3.8)$ \\
\hline Picture-word matching $(/ 20)$ & $19.0(1.6) \dagger$ & $16.0(3.5) \dagger \ddagger$ & $19.8(0.4)$ \\
\hline
\end{tabular}

Mean (SD) values are shown.

*Five patients in this group fell below the 10th percentile score (7) based on normative data from an historical group of 100 healthy controls aged $55-70$ years.

†Significantly worse $(p<0.05)$ than controls.

$¥$ Significantly worse $(p<0.05)$ than the fvFTLD group.

fvFTLD, frontal variant frontotemporal lobar degeneration; MMSE, Mini-Mental State

Examination; NA, not available; tvFTLD, temporal variant frontotemporal lobar degeneration.

T1 weighted volumetric MR images were acquired on a $1.5 \mathrm{~T}$ Signa unit scanner (GE, Milwaukee, USA) using a Spoiled Gradient Echo technique yielding 124 contiguous $1.5 \mathrm{~mm}$ coronal slices. Brain images were processed using MATLAB 7.0 (http://www.mathworks.co.uk) and SPM2 (http://www.fil.ion. ucl.ac.uk/spm/). VBM was performed using a modified optimised protocol and inhouse algorithms, as previously described. ${ }^{12}$ Anatomical regions for small volume correction (based on our prior anatomical hypotheses) comprised right and left inferior and mesial temporal lobes, right and left insula, and bilateral orbitofrontal cortices (see supplementary material available online for details). Scores on the face processing tests for each FTLD subgroup and for the entire FTLD group were entered into linear regression models to investigate associations between test score and grey matter intensity. Performance on tests of face identification and recognition of individual facial emotions (anger, disgust, fear, happiness, sadness, surprise) were analysed in separate models. Voxel intensity was modelled as a function of the test score of interest, covarying for age, gender, total intracranial volume, Mini-Mental State Examination (as an index of overall cognitive function) and face perception score (in order to account for any associated perceptual deficit). Statistical parametric maps (SPMs) for each behavioural contrast were assessed at the whole brain level and after small volume correction using the previously defined anatomical regions at family wise error (FWE) corrected threshold $\mathrm{p}<0.05$.

\section{RESULTS}

Neither the tvFTLD nor the fvFTLD subgroup (analysed separately) showed a VBM association of face or emotion identification performance at the whole brain level or following small volume correction. For the combined FTLD group, SPMs of regional grey matter positively associated with performance on the face and emotion identification tasks are displayed in figure 1 (regional maxima for these SPMs are summarised in supplementary table 2 , available online only). Performance on famous face identification was associated with grey matter volume in the right anterior fusiform gyrus $(\mathrm{p}<0.05$ after FWE correction within the prespecified anatomical small volume). Performance on recognition of anger was associated with grey matter volume in the posterior insula cortex bilaterally $(p<0.05$ after FWE correction within the prespecified anatomical small volume). No other anatomical associations of face identification or individual emotion recognition and no anatomical associations of performance on the face perception task were identified after whole brain or small volume correction at the prescribed threshold. Direct structural contrasts between the tvFTLD and fvFTLD subgroups using VBM did not demonstrate any grey matter voxels overlapping with the anatomical associations of face or emotion identification (even at a lenient statistical threshold of $p<0.001$ uncorrected), suggesting that the behavioural regressions were not driven simply by anatomical differences between subgroups.

\section{DISCUSSION}

Here we have demonstrated structural neuroanatomical substrates for two key dimensions of face processing in a cohort of patients with FTLD. The findings corroborate previous evidence for deficient identification of faces and facial expressions in $\mathrm{FTLD}^{2-6}$ and show that these different components of face processing depend on distinct brain areas. Face identification was associated with grey matter volume in the right fusiform gyrus, somewhat anterior to the fusiform face area (the cortical area previously emphasised as a key site for the abstraction of facial identity information based on focal lesion and normal functional imaging evidence ${ }^{7}$ ). It is unlikely we have demonstrated an anatomical association of face perception per se as the association with face identification performance was present after adjusting for purely perceptual performance. We suggest that this more anterior inferior temporal region linked with face
Figure 1 Statistical parametric maps (SPMs) showing regional grey matter positively associated with performance on famous face identification (left and middle panels) and recognition of angry facial expressions (right panel) in the frontotemporal lobar degeneration (FTLD) cohort. SPMs are displayed on the mean normalised and smoothed T1 weighted MR brain image for the patient group, at a threshold of $p<0.05$ after small volume correction according to face identification
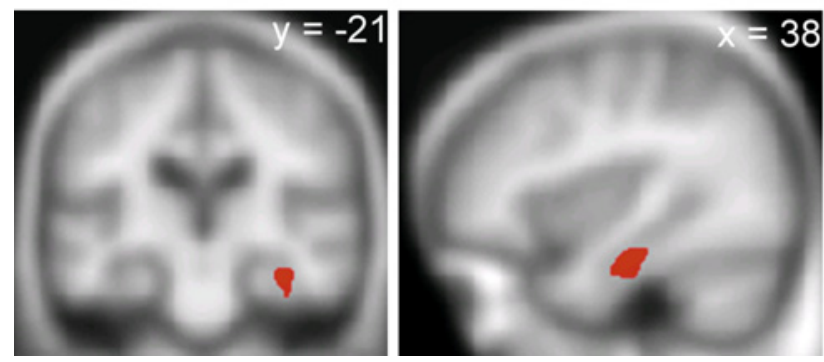
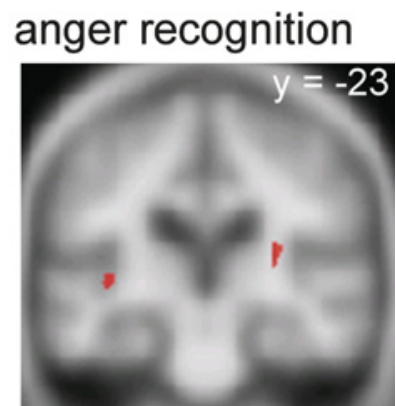
prior anatomical hypotheses. The plane of each section is shown (coordinates in $\mathrm{mm}$, in Montreal Neurological Institute standard stereotactic space); for the coronal sections, the right hemisphere is displayed on the right. 
identification may be involved in semantic memory for faces, consistent both with previous studies of associative prosopagnosia in $\mathrm{FTLD}^{3} 13$ and with functional imaging studies of familiar face processing in normal individuals. ${ }^{7}$

In addition, we have shown an anatomical association for recognition of angry facial expressions in the insula cortex. Anger recognition has previously been identified as a key deficit in FTLD. ${ }^{6}$ Information about brain regions critical for anger recognition is limited but the insula has been implicated previously in the processing of anger by the healthy brain. ${ }^{8}$ The lack of a grey matter association for recognition of other facial expressions may at first appear somewhat surprising in light of present and previous behavioural findings in FTLD. ${ }^{56}$ However, whereas anger is a basic facial expression posing a potential threat to self, the relatively greater conceptual complexity involved in interpreting other facial emotions, such as happiness, fear or disgust, ${ }^{14}$ might make detection of a consistent group level VBM association more difficult. Moreover, VBM work in Huntington's disease has demonstrated an association between insula volume and disgust (but not anger) recognition ${ }^{15}$ : this suggests that the neuroanatomical profile of facial emotion processing may be modulated by the underlying neurodegenerative pathology, and further supports functional imaging evidence in normal subjects that the insula is engaged in processing various emotions according to task demands. ${ }^{8}$ It is also noteworthy that the amygdala was not identified as an association of emotion recognition in our study or in a previous VBM study of FTLD, ${ }^{5}$ which may reflect the relatively low behavioural value and limited arousal potential of the face stimuli for these FTLD patients.

Neuroanatomical associations in a diffuse neurodegenerative disease such as FTLD should be interpreted with care, particularly where (as here) small case numbers limit the strength of those associations. However, this study suggests that neuroanatomical findings concerning face processing mechanisms in FTLD complement focal lesion studies and normal functional imaging work. Like focal lesion studies (and in contrast to normal functional imaging studies), the evidence from neurodegenerative disease can identify brain regions that are critical for particular aspects of face processing. The present findings support cognitive models that posit dissociable processing modules for different aspects of face analysis. ${ }^{16}$ In future work it will be important to study face processing mechanisms using combined behavioural and neuroimaging approaches in a range of neurodegenerative diseases, and to assess the specificity of these mechanisms for processing faces versus other stimuli of comparable perceptual and semantic complexity.

Acknowledgements We are grateful to the subjects for their participation.

Funding This work was undertaken at UCLH/UCL who received a proportion of funding from the Department of Health's NIHR Biomedical Research Centres funding scheme. The Dementia Research Centre is an Alzheimer's Research Trust Co-ordinating Centre. This work was also funded by the Wellcome Trust and the Medical Research Council UK. RO is supported by a Royal College of Physicians/Dunhill Medical Trust Research Fellowship. JDR was supported by a Wellcome Trust Research Training Fellowship and Brain Exit Scholarship. JDW is supported by a Wellcome Trust Senior Clinical Fellowship.

Competing interests None.

Ethics approval This study was conducted with the approval of the the National Hospital for Neurology and Neurosurgery and Institute of Neurology Joint REC.

Provenance and peer review Not commissioned; externally peer reviewed.

\section{REFERENCES}

1. McKhann GM, Albert MS, Grossman M, et al. Clinical and pathological diagnosis of frontotemporal dementia: report of the Work Group on Frontotemporal Dementia and Pick's Disease. Arch Neurol 2001;58:1803-9.

2. Evans JJ, Heggs AJ, Antoun $\mathrm{N}$, et al. Progressive prosopagnosia associated with selective right temporal lobe atrophy. A new syndrome? Brain 1995;118:1-13.

3. Josephs KA, Whitwell JL, Vemuri $P$, et al. The anatomic correlate of prosopagnosia in semantic dementia. Neurology 2008;71:1628-33.

4. Josephs KA, Whitwell JL, Knopman DS, et al. Two distinct subtypes of right temporal variant frontotemporal dementia. Neurology 2009;73:1443-50.

5. Rosen HJ, Wilson MR, Schauer GF, et al. Neuroanatomical correlates of impaired recognition of emotion in dementia. Neuropsychologia 2006;44:365-73.

6. Lough S, Kipps CM, Treise $\mathrm{C}$, et al. Social reasoning, emotion and empathy in frontotemporal dementia. Neuropsychologia 2006;44:950-8.

7. Gobbini MI, Haxby JV. Neural systems for recognition of familiar faces. Neuropsychologia 2007:45:32-41.

8. Phan KL, Wager T, Taylor SF, et al. Functional neuroanatomy of emotion: a meta-analysis of emotion activation studies in PET and fMRI. Neuroimage 2002;16:331-48.

9. Warrington EK, James M. An experimental study of facial recognition in patients with unilateral cerebral lesions. Cortex 1967:3:317-26.

10. Ekman P, Friesen WV. Pictures of facial affect. Palo Alto, CA: Consulting Psychologists Press, 1976

11. Benton AL, Hamsher KS, Varney N, et al. Contributions to neuropsychological assessment: a clinical manual. Oxford: Oxford University Press, 1983.

12. Henley SM, Wild EJ, Hobbs NZ, et al. Defective emotion recognition in early HD is neuropsychologically and anatomically generic. Neuropsychologia 2008;46:2152-60.

13. Snowden JS, Thompson JC, Neary D. Knowledge of famous faces and names in semantic dementia. Brain 2004;127:860-72.

14. Snowden JS, Austin NA, Sembi S, et al. Emotion recognition in Huntington's disease and frontotemporal dementia. Neuropsychologia 2008;46:2638-49.

15. Kipps CM, Duggins AJ, McCusker EA, et al. Disgust and happiness recognition correlate with anteroventral insula and amygdala volume respectively in preclinical Huntington's disease. J Cogn Neurosci 2007;19:1206-17.

16. Bruce V, Young A. Understanding face recognition. Br J Psychol 1986;77:305-27. 